\title{
Correction to: Cabozantinib After a Previous Immune Checkpoint Inhibitor in Metastatic Renal Cell Carcinoma: A Retrospective Multi-Institutional Analysis
}

\author{
Roberto lacovelli ${ }^{1}$. Chiara Ciccarese ${ }^{1,2} \cdot$ Gaetano Facchini $^{3} \cdot$ Michele Milella $^{4} \cdot$ Federica Urbano $^{5} \cdot$ Umberto Basso $^{6}$. \\ Ugo De Giorgi ${ }^{7} \cdot$ Roberto Sabbatini $^{8}$. Daniele Santini ${ }^{9} \cdot$ Rossana Berardi $^{10} \cdot$ Matteo Santoni $^{11} \cdot$ Sergio Bracarda $^{12}$. \\ Francesco Massari ${ }^{13}$. Cristina Masini ${ }^{14}$. Michele De Tursi ${ }^{15} \cdot$ Riccardo Ricotta $^{16}$. Sebastiano Buti ${ }^{17}$. \\ Fable Zustovich ${ }^{18} \cdot$ Pierangela Sepe $^{19} \cdot$ Sabrina Rossetti $^{3} \cdot$ Marco Maruzzo $^{6} \cdot$ Enrico Cortesi $^{5} \cdot$ Giampaolo Tortora $^{1,2}$. \\ Giuseppe Procopio ${ }^{19}$
}

Published online: 28 August 2020

(c) Springer Nature Switzerland AG 2020

\section{Correction to: Targeted Oncology} https://doi.org/10.1007/s11523-020-00732-y

While typesetting the article the Table 3 column/row entries are interchanged. Please find below the updated Table 3.

The original article can be found online at https://doi.org/10.1007/ s11523-020-00732-y.

Roberto Iacovelli

Roberto.iacovelli@policlinicogemelli.it

1 Oncologia Medica, Fondazione Policlinico Universitario Agostino Gemelli IRCCS, Largo Agostino Gemelli 8, 00158 Rome, Italy

2 Oncologia Medica, Facoltà di Medicina e Chirurgia, Università Cattolica del Sacro Cuore, Rome, Italy

3 Departmental Unit of Clinical and Experimental Uro-Andrologic Oncology, Istituto Nazionale Tumori, IRCCS, Fondazione G. Pascale, Naples, Italy

4 Oncologia Medica, Azienda Ospedaliera Universitaria Integrata (AOUI), Verona, Italy

5 Oncology Unit, Department of Radiology, Oncology and Human Pathology, Sapienza University of Rome, Rome, Italy

6 Medical Oncology Unit 1, Department of Oncology, Istituto Oncologico Veneto IOV IRCCS, Padua, Italy

7 Istituto Scientifico Romagnolo per lo Studio e la Cura dei Tumori (IRST) IRCCS, Meldola, Italy

8 Department of Oncology, Hematology and Respiratory Diseases, University of Modena and Reggio Emilia, Modena, Italy
Table 3 Cross-response to nivolumab and cabozantinib

\begin{tabular}{lllll}
\hline $\begin{array}{l}\text { Response to } \\
\text { nivolumab }\end{array}$ & \multicolumn{2}{l}{ Response to cabozantinib } & Total \\
\cline { 2 - 4 } & CR/PR & SD & PD & \\
\hline CR/PR & 9 & 1 & 2 & 12 \\
SD & 14 & 7 & 6 & 27 \\
PD & 15 & 11 & 9 & 35 \\
Total & 38 & 19 & 17 & 74 \\
\hline
\end{tabular}

9 Oncologia Medica, Campus Bio-Medico University of Rome, Rome, Italy

10 Clinica Oncologica, Università Politecnica delle Marche, Azienda Ospedaliero-Universitaria Ospedali Riuniti Umberto I, Ancona, Italy

11 Oncologia Medica, Ospedale di Macerata, Via Santa Lucia 2, 62100 Macerata, Italy

12 S.C. Medical Oncology, Azienda Ospedaliera S. Maria, Terni, Italy

13 Dipartimento di Oncologia Medica, Ospedale Sant'Orsola-Malpighi, Bologna, Italy

14 Medical Oncology Unit, AUSL-IRCCS di Reggio Emilia, Reggio Emilia, Italy

15 Dipartimento di Scienze Orali e Mediche, Sezione di Oncologia, Università G. D’Annunzio, Chieti, Italy

16 Niguarda Cancer Center, Grande Ospedale Metropolitano Niguarda, Milan, Italy

17 Unità Operativa di Oncologia Medica, Azienda Ospedaliero-Universitaria di Parma, Parma, Italy

18 Oncologia Medica, San Martino Hospital, Belluno, Italy

19 Genitourinary Cancer Unit, Dipartimento di Oncologia Medica, Fondazione IRCCS Istituto Nazionale Tumori, Milan, Italy 